\title{
Melioidosis: where do we stand in the development of an effective vaccine?
}

\author{
"It is evident that a number of important knowledge gaps still hinder \\ Burkholderia vaccines from advancing into early phase clinical studies.”
}

\author{
Laura A Muruato' \& Alfredo G Torres ${ }^{*, 1,2}$
}

First draft submitted: 31 January 2016; Accepted for publication: 2 February 2016; Published online: 15 April 2016

\begin{abstract}
Melioidosis is an under-reported neglected tropical disease caused by Burkholderia pseudomallei. Significant worldwide attention has been placed in this disease due to the recent report indicating that the number of estimated cases and fatalities has been significantly under-appreciated. This finding combined with the potential of this bacterium to be used as a biothreat agent has revitalized the research community and the funding agencies to develop and advance to clinical trials an effective vaccine. We present our point of view, based on current scientific evidence, of the actions required to produce an effective vaccine useful for individuals accidentally or purposely exposed to the pathogen and as a way to reduce the number of cases in endemic regions.

Burkholderia pseudomallei is a Gramnegative pathogen and the causative agent of melioidosis, a disease endemic to many tropical and subtropical regions throughout the world [1,2]. Severity of clinical manifestations depends on the route of inoculation and time to treatment. Because of their natural resistance to many commercially available antibiotics, $B$. pseudomallei infections are particularly difficult to treat and
\end{abstract}

often require a cocktail of antibiotics for 12-20 weeks [1,2]. Even with aggressive antibiotic therapy, mortality rates in acute cases approach $40 \%$. In northeast Thailand, human cases of melioidosis are considered the third most common cause of death from an infectious disease (behind HIV and tuberculosis) [3]. Additionally, in northern Australia and regions of southeast Asia, B. pseudomallei infections are a frequent cause of community-acquired pneumonia, with death and septic shock being a common outcome in acute infections [1]. The disease burden in these regions is undeniable, thanks to active disease surveillance. However, disease detection in many areas of the world remains a significant challenge due to the nonspecific clinical manifestations of $B$. pseudomallei infections and the availability of appropriate diagnostics.

Is there currently justifiable need to produce a vaccine? To address this question, we must use a holistic approach to evaluate the overall public health threat of $B$. pseudomallei, both in the USA and worldwide. In a ground-breaking new study, Limmathurotsakul et al. utilized worldwide environmental data and more than 22,000

\section{KEYWORDS}

- antigens • Burkholderia pseudomallei

- immune protection $\bullet$ melioidosis

- vaccine

“...new insights on potential global disease burden provide justification for the importance of developing a vaccine for this under-reported neglected tropical pathogen." 
"Subunit vaccines provide an attractive alternative to live-attenuated strains because of increased safety and potential for largescale production without the need for BSL3 facilities." records of melioidosis infections (human and ani$\mathrm{mal}$ ) to generate a model for predicting the disease burden of melioidosis [4]. Based on this predictive model, the disease is common throughout the tropics, including 34 countries where it has never been reported. Furthermore, this study estimates that in 2015 there were 165,000 cases of melioidosis, 89,000 of which resulted in death [4]. This new model highlights the significant burden of melioidosis around the globe and exposes a great need to increase surveillance and improve pathogen detection in these under-reported areas [5-7]. These new insights on potential global disease burden provide justification for the importance of developing a vaccine for this under-reported neglected tropical pathogen.

Furthermore, because of the high morbidity and mortality, poor response to antibiotic treatment and ease of aerosolization, $B$. pseudomallei has potential for malicious use and, therefore, is classified as a Category B Tier 1 Select Agent. While there is no evidence of $B$. pseudomallei being weaponized, closely related $B$. mallei does have a history of bioweapon use [8]. Meanwhile, inclusion of B. pseudomallei on the Select Agent list has triggered renewed interest and increased financial support to study the disease and accelerate the development of a prophylactic or therapeutic approaches. For example, a vaccine could be used in the event of an intentional or accidental release of the pathogen or to reduce global incidence.

In response to some of these needs, the US Defense Threat Reduction Agency (DTRA) has provided significant financial investments toward the advancement of $B$. pseudomallei and $B$. mallei vaccines. The poor clinical outcomes and possible biothreat applications of these pathogens emphasize the public health ramifications of not having a vaccine. DTRA mandate is to develop new Medical Countermeasures (MCM) and improved animal models to evaluate their effectiveness [9]. The goal of the DTRA program is to support the development of early stage vaccine candidates against these pathogens. The ideal vaccine is one that can generate protective immunity; such candidate(s) are expected to provide vital information regarding the correlates of protective immunity, and must be amenable to large-scale production for the advancement into clinical and preclinical trials.

In the past 3 years, several DTRA-funded vaccine candidates have been tested in preclinical models and significant progress has been made toward understanding what constitutes a protective immune response. However, our knowledge regarding the immune response remains incomplete and impedes progress toward the development of a fully protective vaccine. Although host innate, humoral and cellular immune responses to $B$. pseudomallei infections have been studied for many years, the relationship between the immune response and full protection remains largely undetermined. As a facultative intracellular pathogen, $B$. pseudomallei is capable of invading, replicating and surviving within host cells. Due to its intracellular lifestyle, it is likely that a combination of cell-mediated and humoral responses will be necessary to generate complete protection $[10,11]$. While it is accepted that a strong antibody response is necessary for vaccine-induced protection against $B$. pseudomallei, recent evidence also shows that both $\mathrm{CD} 4^{+}$and $\mathrm{CD} 8^{+} \mathrm{T}$ cells are also important in protection against human melioidosis [12]. Taken together, these findings support the development of vaccine strategies that induce strong humoral and cellular responses.

Various vaccine approaches (live-attenuated, whole-cell killed, subunit, plasmid DNA and dendritic cell vaccines) have been attempted and tested in small animal models with varying success (reviewed elsewhere [3,13]). Live-attenuated vaccines are known to induce a comprehensive immune response by mimicking natural infection and are currently considered the best approach for generating protective immunity against B. pseudomallei and B. mallei [13]. However, Burkholderia live-attenuated strains containing only single gene mutations are not favored because they pose a potential safety risk due to possibility of reversion or persistence [3]. Nevertheless, such live-attenuated strains have been shown to provide significant protection against challenge, and could prove valuable as a platform for continuing vaccine optimization [14,15]. Specifically, the $B$. pseudomallei $2 \mathrm{D} 2$ strain, which contains a mutation in the ilvI gene (encoding an enzyme required for the biosynthesis of branched chain amino acids), was shown to be attenuated in vivo and generated significant protection (up to $100 \%$ ) against wild-type challenge; however, sterilizing immunity was not achieved [15]. Meanwhile, the high efficacy achieved by the 2D2 mutant strain provides the current 'gold standard' by which new live-attenuated strains are measured [16]. While mutants containing deletions of a single gene can provide protection against challenge, the addition of multiple gene mutations often results in a strain that is more attenuated and less likely to revert to 
wild type. Such attenuated strains are important not only for vaccination purposes, but also for the potential to be excluded from Select Agent regulations. Such excluded strains can be studied without the need for high containment facilities, thus providing a valuable tool for broader research applications [17].

Subunit vaccines provide an attractive alternative to live-attenuated strains because of increased safety and potential for large-scale production without the need for BSL3 facilities. However, like live-attenuated vaccines, subunit vaccines are also in need of continuing optimization. While no 'gold standard' for subunit vaccines exists at this time, various subunit vaccine platforms (e.g., glycoconjugates, outer membrane vesicles, etc.) have been shown to generate significant protection against Burkholderia infections. However, it is likely that a multivalent vaccine containing numerous immunogenic epitopes will be necessary to achieve increased vaccine efficacy. The current pool of tested Burkholderia antigens is limited; therefore, there is a need to identify additional antigens and virulence factors that can be incorporated into multivalent vaccines for increased protection. Vaccine optimization for both live-attenuated and subunit vaccines remains a challenge, particularly due to the variability between studies.

In order to accelerate the development of a Burkholderia vaccine, it is important that a consensus be reached regarding a more standardized approach to vaccine testing. To address this need, a Steering Group on Melioidosis Vaccine Development (SGMVD) was recently established to advise research groups and funding agencies regarding the actions needed to advance different vaccine candidates to preclinical studies and beyond [16]. This group outlined the need for standardization of animal models, challenge strains and routes/dosages of vaccination and challenge, as well as the use of proper controls (e.g., using B. pseudomallei 2D2 mutant as positive control). Importantly, all variables were discussed in relation to whether a vaccine would be targeted for biodefense applications or prevention of naturally acquired melioidosis. The SGMVD emphasized the need for head-tohead comparison of the leading candidate vaccines in standardized animal models of infection prior to the advancement to preclinical studies [16].

It is evident that a number of important knowledge gaps still hinder Burkholderia vaccines from advancing into early phase clinical studies. To address these gaps, it is advised that research groups follow SGMVD recommendations in order to more efficiently advance vaccine progression. Standardized methodologies, together with exciting new research being performed on Burkholderia vaccines, will no doubt accelerate the process of obtaining a cost-effective, safe and licensed prophylactic or therapeutic vaccine against melioidosis.

\section{Acknowledgements}

The authors would like to thank Christopher Hatcher for helpful suggestions while writing this editorial.

\section{Financial \& competing interests disclosure}

LA Muruato is supported by the McLaughlin Endowment predoctoral fellowship. The authors have no other relevant affiliations or financial involvement with any organization or entity with a financial interest in or financial conflict with the subject matter or materials discussed in the manuscript apart from those disclosed.

No writing assistance was utilized in the production of this manuscript.

\section{References}

$\checkmark$ Meumann EM, Cheng AC, Ward L, Currie BJ. Clinical features and epidemiology of melioidosis pneumonia: results from a 21-year study and review of the literature. Clin. Infect. Dis. 54, 362-369 (2012).

- 2 Estes DM, Dow SW, Schweizer HP, Torres AG. Present and future therapeutic strategies for melioidosis and glanders. Expert Rev. Anti Infect. Ther. 8, 325-338 (2010).

3 Peacock SJ, Limmathurotsakul D, Lubell Y et al. Melioidosis vaccines: a systematic review and appraisal of the potential to exploit biodefense vaccines for public health purposes. PLoS Negl. Trop. Dis. 6, e1488 (2012).

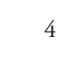

Limmathurotsakul D, Golding N, Dance $\mathrm{DaB}$ et al. Predicted global distribution of Burkholderia pseudomallei and burden of melioidosis. Nat. Microbiol. 1, 15008 (2016).

Doker TJ, Sharp TM, Rivera-Garcia B et al. Contact investigation of melioidosis cases reveals regional endemicity in Puerto Rico. Clin. Infect. Dis. 60, 243-250 (2015).

Currie BJ, Kaestli M. Epidemiology: a global picture of melioidosis. Nature 529, 290-291 (2016).
Corea EM, Merritt AJ, Ler YH, Thevanesam V, Inglis TJ. Sri Lankan National Melioidosis Surveillance Program uncovers a nationwide distribution of invasive melioidosis. Am. J. Trop. Med. Hyg. 292-298 (2016).

${ }_{8}$ Wheelis M. First shots fired in biological warfare. Nature 395, 213 (1998).

9 Defense Threat Reduction Agency. Chemical And Biological Defence Program: Service Call For Proposals. www.dtrasubmission.net/instructions/

Suwannasaen D, Mahawantung J, Chaowagul Wet al. Human immune responses to Burkholderia pseudomallei characterized by 
EDITORIAL Muruato \& Torres

protein microarray analysis. J. Infect. Dis. 203 , 1002-1011 (2011).

- 11 Healey GD, Elvin SJ, Morton M, Williamson ED. Humoral and cell-mediated adaptive immune responses are required for protection against Burkholderia pseudomallei challenge and bacterial clearance postinfection. Infect. Immun. 73, 5945-5951 (2005).

12 Jenjaroen K, Chumseng S, Sumonwiriya $\mathrm{M}$ et al. T-cell responses are associated with survival in acute melioidosis patients. PLoS Negl. Trop. Dis. 9, e0004152 (2015).
Silva EB, Dow SW. Development of Burkholderia mallei and pseudomallei vaccines. Front. Cell. Infect. Microbiol. 3, 10 (2013).

14 Mott TM, Vijayakumar S, Sbrana E, Endsley JJ, Torres AG. Characterization of the Burkholderia mallei ton $B$ mutant and its potential as a backbone strain for vaccine development. PLoS Negl. Trop. Dis. 9, e0003863 (2015).

- 15 Atkins T, Prior RG, Mack K et al. A mutant of Burkholderia pseudomallei, auxotrophic in the branched chain amino acid biosynthetic pathway, is attenuated and protective in a murine model of melioidosis. Infect. Immun. 70, 5290-5294 (2002).

16 Limmathurotsakul D, Funnell SG, Torres AG et al. Consensus on the development of vaccines against naturally acquired melioidosis. Emerg. Infect. Dis. 21, (2015).

17 Federal Select Agent Program. Select Agents And Toxins Exclusions.

www.selectagents.gov/SelectAgentsandToxins 